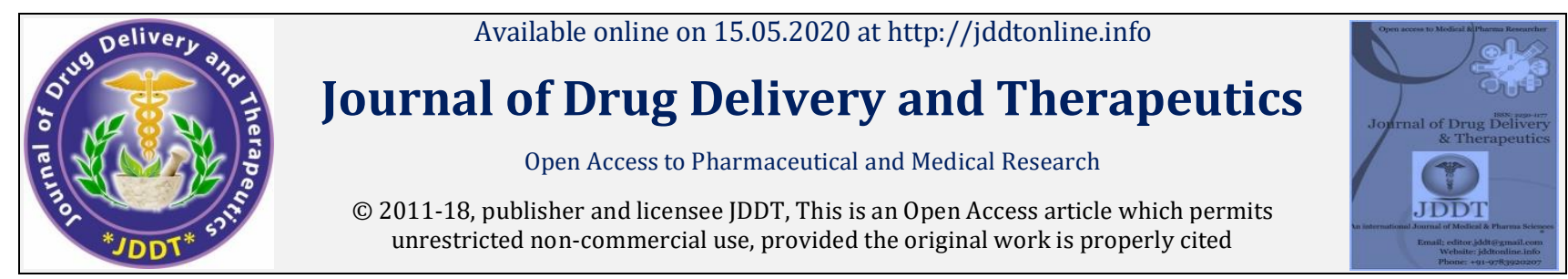

Open Access

Research Article

\title{
Formulation and Evaluation of Pseudoephedrine Hydrochloride Loaded Alginate Microbeads
}

\author{
Dahima Rashmi \\ School of Pharmacy, Devi Ahilya Vishwavidyalaya, Takshashila Campus, Khandwa Road, Indore - 452001, M.P., India
}

\begin{abstract}
Multiple unit dosage forms such as microbeads have increased acceptance because of added even spreading of the drug in the gastrointestinal tract, unvarying drug absorption, abridged local irritation and removal of undesirable intestinal retaining of polymeric material, when compared to non-disintegrating single unit dosage form. The purpose of the presented research is to develop microbeads of pseudoephedrine hydrochloride utilizing sodium alginate as the hydrophilic carrier in combination with HPMC as drug release modifier to lesse n the dosing frequency and thereby advance the patient compliance. The microbeads were formulated by varying concentrations of HPMC and calcium chloride. The optimum formulation was chosen based upon in vitro drug release studies and further evaluated. The compatibility of drugpolymer was studied using FTIR analysis. The prepared formulation underwent evaluation for various parameters like drug entrapment microbeads size, swelling index, mucoadhesive property and stability. No significant drug-polymer interactions were observed in compatibility studies and the formulation was found to be stable on 45 days storage. The formulations exhibited an extended drug release pa ttern which was the ultimate aim of the study. The microbeads represented good yield, high drug entrapment, low microbeads size and appropriate swelling property. The in vitro wash-off test indicated that the sodium alginate microbeads represent decent mucoadhesive properties. Henceforth, the formulated HPMC coated sodium alginate beads can be utilized as a substitute and cost-effective carrier for the oral controlled delivery of pseudoephedrine hydrochloride.
\end{abstract}

Keywords: microbeads, pseudoephedrine hydrochloride, sodium alginate, drug release

Article Info: Received 18 March 2020; $\quad$ Review Completed 24 April 2020; $\quad$ Accepted 30 April 2020; Available online 15 May 2020

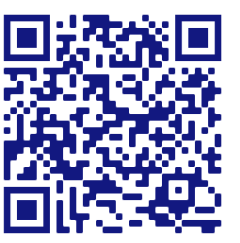

Cite this article as:

Dahima R, Formulation and Evaluation of Pseudoephedrine Hydrochloride Loaded Alginate Microbeads, Journal of Drug Delivery and Therapeutics. 2020; 10(3):137-141 http://dx.doi.org/10.22270/jddt.v10i3.4094

*Address for Correspondence:

Dr. Rashmi Dahima, School of Pharmacy, Devi Ahilya Vishwavidyalaya, Takshashila Campus, Khandwa Road, Indore -

452001, M.P., India.

\section{INTRODUCTION}

Oral drug delivery is the furthermost required and ideal way of administering therapeutic candidates for their systemic effects. Furthermore, the oral medication is largely measured as the initial path explored in the discovery and advancement of novel therapeutic entities and pharmaceutical preparations, chiefly owing to patient reception, ease and cost-effective manufacturing procedure 1. For ample drug candidates, controlled drug delivery system delivers clinically active cure while preserving the essential balance of pharmacokinetic and pharmacodynamic outlines with satisfactory level of safety to the patient 2 . A controlled release system comprises any conveyance arrangement that attains deliberate release of the drug over a prolonged period of time, guarantees safety, efficiency of the drugs and patient compliance. Effective commercialization of a controlled release formulation is often challenging and comprises attention to many issues such as physiochemical properties of the drug, physiological factors and manufacturing variables ${ }^{3}$.

Controlled drug delivery system holds promising results in site-specific targeting. One of such approach is the formulation of microbeads as drug carriers. Multiple unit dosage forms such as microspheres or microbeads have increased acceptance because of added even spreading of the drug in the gastrointestinal tract, more unvarying drug absorption, abridged local irritation and removal of undesirable intestinal retaining of polymeric material, when compared to non-disintegrating single unit dosage form 4 Microbeads are small, solid and free flowing particulate carriers encompassing dispersed drug particles either in solution or crystalline form that permits a sustained release or multiple release profiles of treatment with various active agents without major side effects ${ }^{5}$.

Inotropic gelation method for the preparation of microbeads is grounded on the electrostatic interaction amongst polymer's amino cluster and negatively charged assembly of 
polyanion. Natural polysaccharides are used as biopolymers in novel drug delivery system, thus ensuring eco-friendly pharmaceutical development. Amongst the natural polymers, sodium alginate has been considered typically and generates reticulated assembly when cross-linked with polyvalent or divalent ions 6. Pseudoephedrine hydrochloride is a sympathomimetic drug efficacious for treating nasal congestion and vasoconstriction. Biological half-life $\left(t_{1 / 2}\right)$ of pseudoephedrine hydrochloride is less, furthermore it is sparingly water-soluble drug and exhibits a low dissolution rate. Pseudoephedrine hydrochloride when formulated in a dosage from such as tablets reveals duration of action of 3 to $4 \mathrm{~h}$, whereas when formulated as extended release form exhibit duration of action of 8 to $12 \mathrm{~h} 7$; hence, it was thought worth to fabricate one such sustained release form of pseudoephedrine hydrochloride as microbeads.

The aim of the presented research is to develop sustained release oral product namely microbeads of pseudoephedrine hydrochloride using sodium alginate as the hydrophilic carrier in combination with HPMC as drug release modifier to reduce the dosing frequency and thereby improve the patient compliance. Also, to improve its bioavailability by bypassing the first pass metabolism because alginate beads shrink and unable to swell at acidic environment and the encapsulated drugs are not released whereas they easily swell in an alkaline environment and release the drug.

\section{MATERIALS AND METHODS}

\section{Preparation of alginate microbeads}

Pseudoephedrine hydrochloride microbeads were prepared by dissolving sodium alginate in distilled water at $800 \mathrm{rpm}$ for $30 \mathrm{~min}$ to get a bubble free clear solution $(2 \% \mathrm{w} / \mathrm{v})$. Drug and polymer (HPMC) were taken (Table 1) and dispersed in sodium alginate solution and mixed thoroughly to form a smooth viscous dispersion. Resulted dispersion was dropwise added to calcium chloride solution $(5 \% \mathrm{w} / \mathrm{v}$ in distilled water), through a needle of 20-gauge size with continuous stirring at the speed of $500 \mathrm{rpm}$. The cations diffuse into the drug loaded polymeric drops, forming a three-dimensional lattice of ionically cross-linked moiety. The resulting microbeads were collected by filtration and washed repeatedly with purified water to remove excess calcium deposited on the surface of microbeads. The resultant microbeads were dried under vacuum.

\section{Product yield}

The yield of the prepared formulations was calculated as the percentage of the weight of the dried product at room temperature compared to the theoretical amount. Production yield is calculated using the following equation:

Percentage yield $=$ [weight of product $/$ (weight of drug + polymer)] $\times 100$

\section{Drug entrapment efficiency}

Pseudoephedrine hydrochloride content in microbeads was estimated by UV-spectrophotometric method. Accurately weighed (50 mg) microbeads samples were added in $100 \mathrm{ml}$ of phosphate buffer (pH 7.4) and kept for stirring for $24 \mathrm{~h}$. The solution was filtered and the free drug concentration was determined spectrophotometrically at $257 \mathrm{~nm}$ using UVvisible spectrophotometer. The entrapment efficiency for all the formulations was calculated by using following formula:

Percent entrapment efficiency $=$ (estimated drug content/theoretical drug content) $\times 100$

\section{Optimization}

Various formulation variables e.g. drug concentration, polymer concentration, emulsifier concentration, concentration of cross-linking agent and process variables viz. stirring speed were identified and studied (Table 1). In order to get optimized product dissolution studies were carried out.

Table 1: Variables applied for optimization of microbeads formulation.

\begin{tabular}{|c|c|c|c|c|c|c|}
\hline \multirow{2}{*}{ S. No. } & Parameter & \multicolumn{5}{|c|}{ Formulation } \\
\cline { 2 - 7 } & & $\mathbf{A 1}$ & $\mathbf{A 2}$ & $\mathbf{A 3}$ & $\mathbf{A 4}$ & A5 \\
\hline 1 & Drug & 1.0 & 1.0 & 1.0 & 1.0 & 1.0 \\
\hline 2 & HPMC & 0.5 & 1.0 & 1.5 & 1.0 & 1.0 \\
\hline 3 & Sodium alginate & 2.0 & 2.0 & 2.0 & 2.0 & 2.0 \\
\hline 4 & Calcium chloride & 5.0 & 5.0 & 5.0 & 3.0 & 7.0 \\
\hline
\end{tabular}

\section{In vitro drug release studies}

The in vitro drug release studies of drug loaded microbeads were carried out using USP dissolution apparatus type I in different mediums. Microbeads were weighed $(100 \mathrm{mg})$ and gently spread over the surface of $900 \mathrm{ml}$ of dissolution medium. The content was rotated at $50 \mathrm{rpm}$ at $37 \pm 0.5{ }^{\circ} \mathrm{C}$. Perfect sink condition was maintained during drug dissolution study period. The simulation of gastrointestinal transit condition was achieved by altering the $\mathrm{pH}$ of dissolution medium at different time intervals. The $\mathrm{pH}$ of dissolution medium was maintained at 1.2 for $2 \mathrm{~h}$ using 0.01 $\mathrm{N} \mathrm{HCl}$. The medium was filtered through membrane filter $(0.45 \mu \mathrm{m})$, after few hours when the sample were withdrawn the residue on filter paper was added to the next medium immediately, that is phosphate buffer $\mathrm{pH} 7.4$ and the samples $(10 \mathrm{ml})$ were withdrawn at particular time intervals of $15 \mathrm{~min}$ from another medium as well. The samples were analysed using UV-visible spectrophotometer at $257 \mathrm{~nm}$ for drug release. The receptor volume was maintained constant by replacing with equivalent volume of medium after each withdrawal. The drug concentration was calculated using regression equation of the calibration curve.

\section{Drug-polymer compatibility study in microbeads}

Compatibility studies in optimized formulation were performed using Fourier Transform infrared (FTIR) spectroscopic analysis. To check the compatibility of the pseudoephedrine hydrochloride with polymer, IR spectra of drug, polymer and combination of the drug and polymer were taken on an FTIR spectrophotometer in the wave number region of 400 and $4000 \mathrm{~cm}^{-1}$.

Measurement of microbeads size by optical microscopy 
Size of the prepared microbeads in optimized formulation was determined using an optical microscope fitted with a stage and an ocular micrometre. Mean diameter was calculated by measuring diameter of $50 \mathrm{mg}$ dried microbeads of formulation.

\section{Swelling index study}

The extent of swelling was measured in terms of percent weight gain by the beads. In this test $20 \mathrm{mg}$ of beads was kept in petri dish containing phosphate buffer ( $\mathrm{pH}$ 7.4). At the end of $1 \mathrm{~h}$, the beads were withdrawn, soaked with tissue paper and weighed. Then for every $1 \mathrm{~h}$, weights of beads were noted and the process was continued till the end of $8 \mathrm{~h}$. The percent weight gain by the beads was calculated by the following formula:

Swelling index $(\mathrm{SI})=\left[\left(\mathrm{W}_{\mathrm{t}}-\mathrm{W}_{0}\right) / \mathrm{W}_{0}\right] \times 100$

Where, $\mathrm{W}_{\mathrm{t}}=$ mass of swollen beads at time $\mathrm{t}, \mathrm{W}_{0}=$ mass of dry beads at $\mathrm{t}=0$

\section{Mucoadhesive test}

The mucoadhesive property of microbeads was evaluated by an in vitro adhesion testing method known as wash-off method. Freshly excised pieces of chicken intestinal mucosa were mounted on to glass slides with cotton thread. About 20 microbeads were spread on to each prepared glass slide and immediately thereafter the slides were hung to USP II tablet disintegration test apparatus. When the test apparatus was operated, the sample is subjected to slow up and down movement in the test fluid at $37{ }^{\circ} \mathrm{C}$ contained in a one litre vessel of the apparatus. At an interval of $30 \mathrm{~min}$ up to $8 \mathrm{~h}$, the machine was stopped and number of beads still adhering to mucosal surface was counted. The test was performed at intestinal (phosphate buffer pH 7.4) condition.

\section{Stability studies}

To assess the long-term physical stability, microbeads from were filled into a hard gelatin capsules manually and wrapped the filled capsule with aluminium foil and entire packet was kept in to self-sealing polyethylene cover. The stability studies were performed at room temperature for period of 45 days. Particle size studies (with optical microscope) and drug content analysis (spectrophotometrically at $257 \mathrm{~nm}$ ) were conducted every 7 days for the entire period of stability study.

\section{RESULTS AND DISCUSSION}

Chemical reaction between sodium alginate and calcium chloride to form calcium alginate was utilized for the microencapsulation of pseudoephedrine hydrochloride core material. For slowing the drug release hydrophilic polymers were added in different concentration so that the drug will release constantly for $24 \mathrm{~h}$. The yield of all the formulations was found to be in the range of $77.23 \pm 0.35$ to $89.87 \pm$ $0.46 \%$. The drug entrapment efficiency determines the percentage of encapsulated drug with respect to the total drug introduced into polymer solution. Our study indicated that the drug entrapment efficiency for various formulations of pseudoephedrine hydrochloride in microbeads was found to be in range of $76.30 \pm 0.38$ to $92.14 \pm 0.54 \%$ (Table 2).

Table 2: Percentage yield and drug entrapment efficiency of various microbeads formulation of pseudoephedrine hydrochloride $(n=3)$.

\begin{tabular}{|c|c|c|}
\hline $\begin{array}{c}\text { Formulation } \\
\text { Percentage Yield } \\
\text { (Mean } \pm \text { S.E.M.) }\end{array}$ & $\begin{array}{c}\text { Drug Entrapment Efficiency } \\
\text { (Mean } \pm \text { S.E.M.) }\end{array}$ \\
\hline A1 & $77.23 \pm 0.35$ & $86.23 \pm 0.51$ \\
\hline A3 & $86.90 \pm 0.50$ & $92.14 \pm 0.54$ \\
\hline A4 & $89.87 \pm 0.46$ & $80.75 \pm 0.46$ \\
\hline A5 & $79.34 \pm 0.41$ & $76.30 \pm 0.38$ \\
\hline
\end{tabular}

It is obvious that increasing calcium chloride concentration produces beads with higher levels of $\mathrm{Ca}^{2+}$ ions. Consequently, the cross-linking of the polymer and compactness of the formed insoluble dense matrices also increases, resulting in more drug entrapment in the microbeads. On other hand further increase in the concentration of calcium chloride above $(5 \% \mathrm{w} / \mathrm{v})$ do not enhance the drug loading. This could be due to possible saturation of calcium binding sites in the guluronic acid chain, preventing further $\mathrm{Ca}^{2+}$ ions entrapment and, hence, cross-linking was not altered with higher concentration of calcium chloride solution ${ }^{8}$. Therefore, the percentage of calcium chloride in our study was chosen in such a manner to get maximum drug loading efficiency.

Dissolution studies were carried out for formulation optimization. Firstly, we observed the dissolution profile of prepared microbeads in $0.01 \mathrm{~N} \mathrm{HCl}$ and phosphate buffer $(\mathrm{pH}$ 7.4) by varying the concentration of polymer (HPMC) and keeping rest of the excipients unchanged. Dissolution rate was found out to be increased with an increase in time. Amongst formulations with various drug polymer ratio viz. $1: 0.5,1: 1$ and $1: 1.5$, the formulation with $1: 1$ ratio exhibited best drug release, hence optimized for other preparations (Figure 1). 
(A) Dissolution in $\mathrm{HCl}$

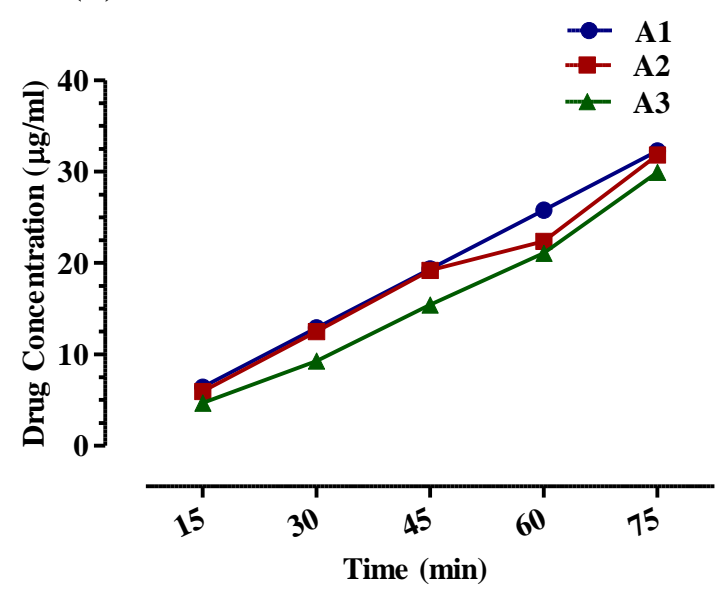

(B) Dissolution in Phosphate Buffer

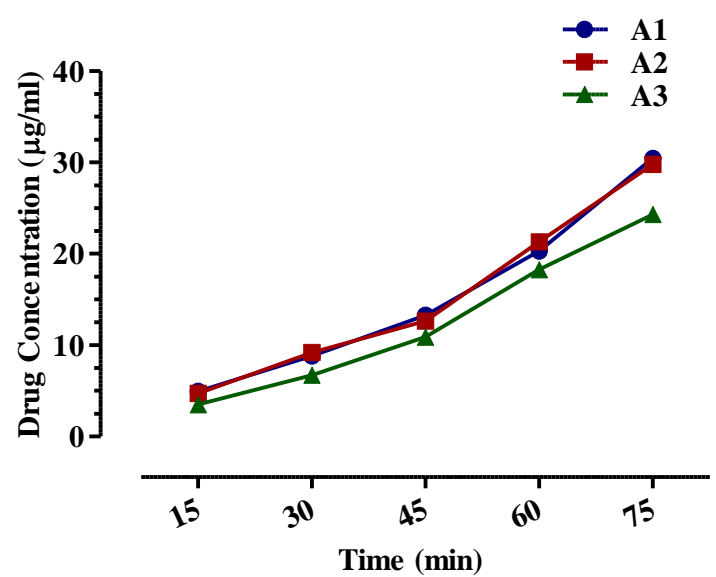

Figure 1: In vitro drug release studies for pseudoephedrine hydrochloride loaded alginate microbeads prepared using varying concentration of HPMC.

Calcium chloride is mainly used for crosslinking. We used varying concentrations $(3 \%, 5 \%$ and $7 \% \mathrm{w} / \mathrm{v})$ of calcium chloride in our previously optimized formulation. It was found that, at lower concentration (3\%) dissolution increased whereas at higher concentration (7\%), dissolution decreased. Hence, the best results were obtained with $5 \%$ calcium chloride (Figure 2). Hence, the formulation A2 with drug:polymer:sodium alginate:calcium chloride as 1:1:2:5, has been optimized and utilized for further studies.

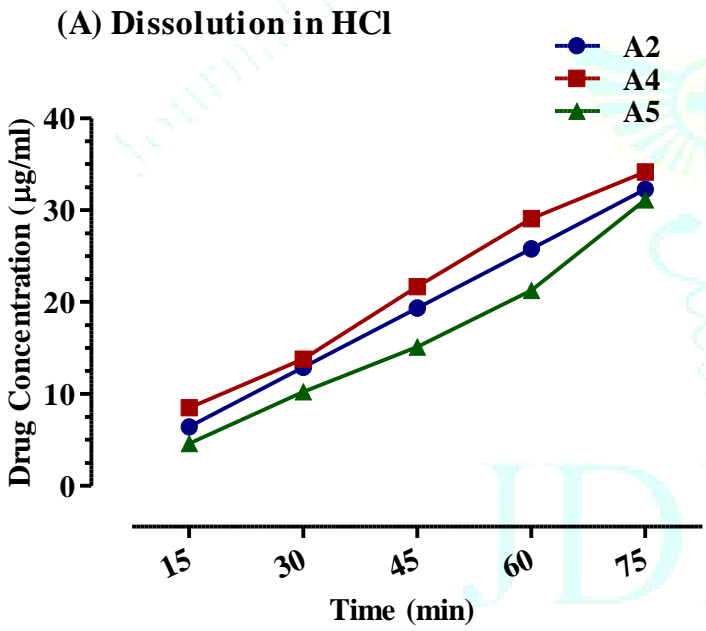

\section{(B) Dissolution in Phosphate Buffer}

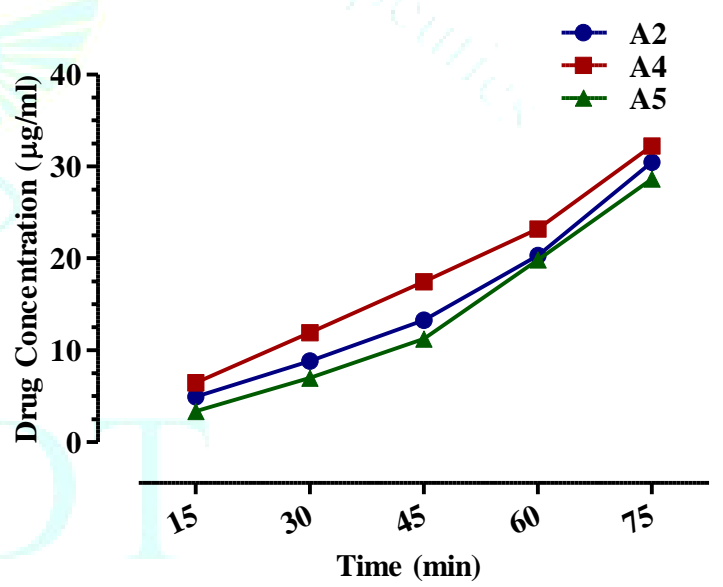

Figure 2: In vitro drug release studies for pseudoephedrine hydrochloride loaded alginate microbeads prepared using varying concentration of calcium chloride.

The compatibility of pseudoephedrine hydrochloride with various polymers was investigated by FTIR-spectroscopy study. The IR spectra of the drug and polymer combination were compared with the spectra of the pure drug. In which no shifting of peaks was significantly found, indicating the stability of the drug during encapsulation process. The mean particle size of optimized formulation of pseudoephedrine hydrochloride microbeads was found to be $867.45 \pm 3.08 \mu \mathrm{m}$. It has been specified that when a drop of alginate solution comes in contact with calcium ions, gelation occurs instantaneously. As $\mathrm{Ca}^{+2}$ ions, penetrates into interior of droplets, water is squeezed out of the interior of droplets resulting in contraction of beads ${ }^{9}$. The size of the spherical matrix could easily be controlled by varying the stirring speed and cross-linking time of the system. The swelling behaviour of the beads was studied by measuring the weight of the beads after exposure to phosphate buffer, pH 7.4 for 8 h. Degree of swelling is proportional to ratio of drug to polymer and it affects the property of drug release from polymer. The mean swelling index of optimized formulation of pseudoephedrine hydrochloride microbeads was found to be $62.35 \pm 0.14 \%$ (Table 3 ). Under neutral conditions the beads swell and the drug release depends on the swelling and erosion process. Being a polyelectrolyte, alginate can exhibit swelling properties that are sensitive to the $\mathrm{pH}$, ionic strength and ionic composition of the medium 10. The optimized batch was subjected to mucoadhesive test and formulation A2 exhibited $80.71 \pm 0.55 \%$ mucoadhesive property. The optimized formulation was subjected to stability studies at room temperature 45 days. Thereafter, the beads size and drug content were determined. Results from the stability studies indicated that beads were physically and chemically stable for more than 45 days, exhibiting no significant change in bead size and drug content. 
Table 3: Evaluation parameters of alginate microbeads formulation of pseudoephedrine hydrochloride

\begin{tabular}{|c|c|c|c|}
\hline \multirow{2}{*}{ Optimized Batch } & Microbeads size $(\boldsymbol{\mu m})$ & Swelling index (\%) & Mucoadhesion (\%) \\
\cline { 2 - 4 } & \multicolumn{3}{|c|}{ Mean \pm S.E.M. (n=3) } \\
\hline A2 & $867.45 \pm 3.08$ & $62.35 \pm 0.14$ & $80.71 \pm 0.55$ \\
\hline
\end{tabular}

\section{CONCLUSION}

Pseudoephedrine hydrochloride microbeads were successfully prepared by ionotropic gelation method. Among the batches, A2 exhibited better drug release and further used to determine the microbead size, swelling index, mucoadhesive property and stability. All the evaluated parameters found to be in promising range. Therefore, it can be assumed that the pseudoephedrine hydrochloride microbeads are promising pharmaceutical dosage forms, which provide sustained release drug delivery system and improve bioavailability. The entire process is feasible in an industrial scale and can be applied for further pilot study.

\section{CONFLICT OF INTEREST}

The author does not have any conflict of interest.

\section{REFERENCES}

1. Sherina VM, Santhi K, Sajeeth CI, "Formulation and evaluation of sodium alginate microbeads as a carrier for the controlled release of nifedipine" IJPCS, 2012; 1(2):699-710.

2. Manjanna KM, Shivakumar B, Pramodkumar TM, "Diclofenac sodium microbeads for oral sustained drug delivery" Int J Pharm Tech Res, 2009; 1(9):317-327.
3. Lachman L, Lieberman HA, Kanig JL. The theory and practice of industrial pharmacy. $3^{\text {rd }}$ ed. Mumbai: Varghese Publishing House; 1987. P. 230.

4. Malik RK, Malik P, Gulati N, Nagaich U, "Fabrication and in vitro evaluation of mucoadhesive ondansetron hydrochloride beads for the management of emesis in chemotherapy" Int J Pharm Invest, 2013; 3(1):42-46.

5. Menon TV, Sajeeth CI, "Formulation and evaluation of sustained release sodium alginate microbeads of carvedilol" Int J Pharm Tech Res, 2013; 5(2):746-753.

6. Mattam J, Sailaja K, "Preparation and evaluation of sulfasalazine loaded sodium alginate microbeads for sustained delivery" AJPCR, 2016; 9(2):66-70.

7. Joe VF, Prasanth VV, Viswanath BA et al., "Formulation and evaluation of pseudoephedrine hydrochloride and loratadine extended release tablet" J Pharm Res, 2011; 4(2):507-508.

8. Rastogi R, Sultan Y, Aqil M, "Alginate microspheres of isoniazid for oral sustained drug delivery" Int J Pharm, 2007. 334(1-2):71-77.

9. Pralhad TT, Rajendra Kumar DK, "Encapsulation of waterinsoluble drugs by a cross-linking technique" AAPS Pharm Sci, 2004; 6(12):1-8.

10. Sevgi T, Omer HO, Fusun A, "Formulation and investigation of nicardipine $\mathrm{HCl}$-alginate gel beads with factorial design-based studies" Euro J Pharm Sci, 1998; 6(3):241-246. 